
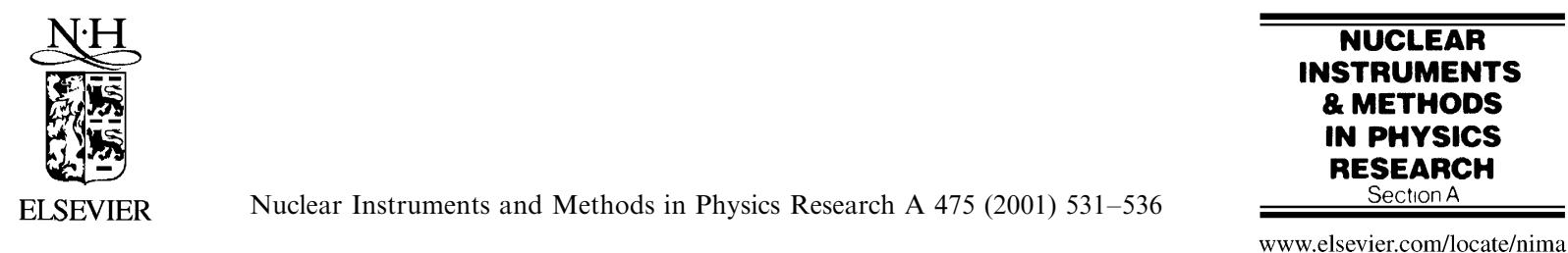

\title{
Transient mirror heating theory and experiment in the Jefferson Lab IR Demo FEL
}

\author{
S. Benson*, Michelle Shinn, G.R. Neil \\ Jefferson Lab, MS 6A, 12000 Jefferson Ave., TJNAF, Newport News, VA 23606, USA
}

\begin{abstract}
During commissioning of the IR Demo FEL at Jefferson Lab, we noticed that the FEL exhibited a rapid power drop with time when the first set of $3 \mu \mathrm{m}$ mirrors was used. Though the rate of power drop was unexpected, it was thought that it could be due to a distortion of the mirrors during a time short compared to the thermal diffusion time. This transient distortion might affect the laser more than the steady state distortion. This paper presents some analysis of the transient mirror heating problem and some recent experimental results using different mirror substrates and coatings. It is found that the behavior of the first mirror set cannot be reconciled with the observed power falloff if a linear absorption is assumed. The power drop in more recent experiments is consistent with linear thermal analysis. No anomalous transient effects are seen. (C) 2001 Elsevier Science B.V. All rights reserved.
\end{abstract}

PACS: 41.60.Cr; 42.60.Da

Keywords: High power; Resonators; Optics

\section{Introduction}

In the previous work [1] we described the steady state behavior of a near-concentric resonator with losses only at the output coupler. Subsequent measurement of the distortion induced using a $\mathrm{CO}_{2}$ laser agreed with the distortion predicted by that paper. It was also found that the power of the IR Demo laser saturated at a value similar to that predicted by that theory when a resonator with $\mathrm{CaF}_{2}$ mirrors was used [2]. During initial attempts to lase at high power with sapphire mirrors we found that the laser saturated at a very low power

\footnotetext{
*Corresponding author. Tel.: +1-757-269-5026; fax: +1757-269-5519.

E-mail address: felman@jlab.org (S. Benson).
}

and that the power dropped extremely rapidly from the kilowatt level down to a few hundred watts (see Fig. 7 from Ref. [2]). This behavior motivated us to study the transient heating effects in a high power FEL. The optical cavity parameters for the IR Demo FEL are given in Table 1. The magnification $M$, defined as the ratio of the mode size on the mirrors to the mode size at the waist in the cavity, was chosen to be 101 as a compromise between mirror heating and resonator stability issues.

The design details of the accelerator have been reported previously in Ref. [3]. The accelerator can deliver up to $240 \mathrm{~kW}$ of continuous electron beam power. The laser efficiency is up to $1.0 \%$ at full power and $1.5 \%$ at low power. With a $40 \mathrm{~cm}$ Rayleigh range and $10 \%$ output coupling, the CW 
Table 1

Design parameters for the IR Demo optical resonator

\begin{tabular}{ll}
\hline Parameter & Value \\
\hline Length & $8.0105 \mathrm{~m}$ \\
Mirror radius of curvature & $404.5 \mathrm{~cm}$ \\
Rayleigh range & $40 \mathrm{~cm}$ \\
Magnification & 101 \\
Mirror diameter & $5 \mathrm{~cm}$ \\
Mirror tilt tolerance & $1.5 \mu \mathrm{rad} \sqrt{\lambda(\mu \mathrm{m})}$ \\
Typical output coupler reflectivity & $90 \%$ \\
HR reflectivity & $>99.5 \%$ \\
Coating absorption & $<0.1 \%$ \\
\hline
\end{tabular}

intensity at the cavity center could be as high as $2.8 \mathrm{MW} / \mathrm{cm}^{2}$. With a magnification of 101 the intensity at the mirrors is $28 \mathrm{~kW} / \mathrm{cm}^{2}$. The gain is difficult to measure but is of the order of $100 \%$ per pass as judged from the turn-on time and the dependence of the efficiency versus number of round trips per gain pass.

\section{Steady state mirror distortion}

In Ref. [1] it was shown that the mirror distortion in the case of a Gaussian absorption pattern on a mirror whose edge is held at a constant temperature has the form

$$
\begin{aligned}
\delta z(\chi)= & \frac{P_{\ell}}{8 \pi F}\left[\gamma+\ln \left(\frac{2 a^{2}}{w_{\mathrm{m}}^{2}}\right)-1.17 R_{20}(\chi)\right. \\
& \left.+0.44 R_{40}(\chi)-0.17 R_{60}(\chi)\right]
\end{aligned}
$$

where $P_{\ell}$ is the output laser power, $a$ is the mirror radius, $w_{\mathrm{m}}$ is the $1 / \mathrm{e}^{2}$ laser mode radius on the mirror, $R_{\mathrm{mn}}$ is the Zernike circle polynomial of radial order $m$ and azimuthal order $n$, and $\gamma$ is the Euler-Mascheroni constant, equal to 0.57722. The quantities $F$ (the figure of merit for the mirror) and $\chi$ are defined by

$\chi=\frac{r}{2 w_{\mathrm{m}}} \quad$ and $\quad F=\frac{k_{\mathrm{th}}}{\left(h \alpha_{\mathrm{B}}+\alpha_{\mathrm{s}}\left(1+1 / t_{\mathrm{c}}\right)\right) \alpha_{\mathrm{e}}}$

where $k_{\mathrm{th}}$ is the thermal conductivity of the mirror substrate, $h$ is the mirror thickness, $t_{\mathrm{c}}$ is the output coupler transmission, $\alpha_{\mathrm{B}}$ is the bulk absorption coefficient, $\alpha_{\mathrm{s}}$ is the coating absorption, and $\alpha_{\mathrm{e}}$ is the thermal expansion coefficient of the mirror substrate. The quantity in parentheses in the denominator of the expression for $F$ is the total absorption of the mirror. From this solution, it is possible to calculate the change in the Rayleigh range and the aberration for a given set of mirrors, laser wavelength, and power output. In Ref. [1] we assumed that only one mirror suffered distortion. To better model the IR Demo performance, we assume here that both mirrors have equal distortion. With this assumption, the change in the Rayleigh range is given by

$\frac{\Delta z_{\mathrm{R}}}{z_{\mathrm{R}}} \cong \frac{1.17 P_{\ell}}{16 F \lambda} \frac{M}{\sqrt{M-1}}$.

Note that the ultimate change in the Rayleigh range will be larger than the value calculated by this formula when the change is large due to the positive feedback of the mirror heating. As an example, for the IR Demo a $60 \%$ initial change will lead to a $100 \%$ change when the change is calculated self-consistently.

The aberration may be calculated from the higher order Zernike polynomial coefficients in Eq. (1). The resulting equation can be rewritten in terms of the change in the Rayleigh range:

$\frac{\delta z(0)}{\lambda}=0.332 \frac{\sqrt{M-1}}{M} \frac{\Delta z_{\mathrm{R}}}{z_{\mathrm{R}}}$.

For large magnification and moderate changes in the Rayleigh range, the aberration is much smaller than one wave. For a magnification of 101 and a $100 \%$ change in the Rayleigh range, the aberration is only $3 \%$ of a wave.

For very large changes in the Rayleigh range, or smaller magnifications, the aberration may limit the laser power.

\section{Transient mirror distortion}

When the laser first turns on there is a period during which the energy deposited in the mirrors has not had a chance to spread laterally. The mirror distortion should therefore have the shape of the optical mode instead of the shape given in Eq. (1). This transient regime is present for times short compared to the time necessary for the heat 
to spread laterally by a mode radius, given by

$t_{0}=\frac{w_{\mathrm{m}}^{2}}{8 \kappa}$

where $\kappa$ is the thermal diffusivity of the mirror substrate. Note that, if the mirrors distort in such a way to change $w_{\mathrm{m}}$, the characteristic time $t_{0}$ changes as well. The temperature rise of a half plane on which a Gaussian mode is incident is given by the following equation [4]:

$$
\begin{aligned}
& T(r, z, t)=\frac{2 P}{\rho C(4 \pi \kappa)^{3 / 2}} \\
& \times \int_{0}^{t} \frac{1}{\left(t^{\prime}+t_{0}\right) \sqrt{t^{\prime}}} \exp \left[\frac{-z^{2}}{4 \kappa t^{\prime}}-\frac{r^{2}}{4 \kappa\left(t^{\prime}+t_{0}\right)}\right] \mathrm{d} t^{\prime} .
\end{aligned}
$$

The distortion versus time may be calculated by integrating Eq. (6) along the $z$ coordinate. The integral over $t$ may then also be carried out. When the resulting equation is multiplied by the expansion coefficient we get

$$
\begin{aligned}
& \alpha_{\mathrm{e}} \int_{0}^{\infty} T(r, z, t) \mathrm{d} z \\
& =\frac{P_{\mathrm{abs}} \alpha_{\mathrm{e}}}{4 \pi k_{\mathrm{th}}}\left[\operatorname{Ei}\left(\frac{r^{2}}{4 \kappa\left(t_{0}+t\right)}\right)-\operatorname{Ei}\left(\frac{r^{2}}{4 \kappa t_{0}}\right)\right]
\end{aligned}
$$

where Ei is the tabulated exponential integral [5]. For times small compared with $t_{0}$ the expression in brackets can be approximated by the Gaussian mode shape at the mirror. For large times, the term in brackets approaches Eq. (1). The distortion at the mode center versus time is given by

$\delta z(t)=\frac{P_{\mathrm{abs}} \alpha_{\mathrm{e}}}{4 \pi k_{\mathrm{th}}} \ln \left(1+\frac{t}{t_{0}}\right)$.

For small times, the Zernike coefficients will be different from those in Eq. (1). If the Gaussian mode is expanded in the same Zernike polynomials, the coefficients are

$$
\begin{gathered}
c_{1}=-0.283, \quad c_{2}=0.275, \quad c_{3}=-0.186, \\
\text { and } \quad c_{4}=0.110 .
\end{gathered}
$$

If these are compared with those of Eq. (2), it is found that the aberration amplitude is about the same as for the steady state case. The curvature coefficient is smaller by a factor of 4 . This means that the relative importance of radius of curvature effects and aberrations may be reversed for transient versus steady state operation. It is likely that aberrations are always the limiting factor for transient distortion while they are only a factor in steady state for cavities with small magnification.

Though we can calculate the change in the aberrations and Rayleigh range from a given power absorption on a mirror, it is difficult to predict how this distortion affects the laser. The most definitive way to determine the effect is to do a full two-dimensional simulation that includes the mirror distortion and cavity phase advances and calculates the saturated power. This can be done by a simulation code such as FELIX, developed at Los Alamos. Modeling a deep UV system using FELIX [6] found that the power drops rapidly with increasing wavefront distortion for a small mirror surface distortion per unit intensity. Let us define the mirror surface distortion per unit incident intensity as $\Delta$. The quantity $\Delta$ is a measure of the mirror coating absorption or the expansion coefficient of the mirror substrate. For large values of $\Delta$, the circulating power falls off sufficiently fast to keep the total wavefront distortion of the circulating mode constant with respect to $\Delta$. This is shown in Fig. 1. It can also be a function of the repetition rate if one assumes that the right axis is the power per micropulse instead of the total power. Finally, the power versus time will be similar to the power versus $\Delta$ as long as the distortion is linearly dependent on time. The latter assumption is true during the transient regime.

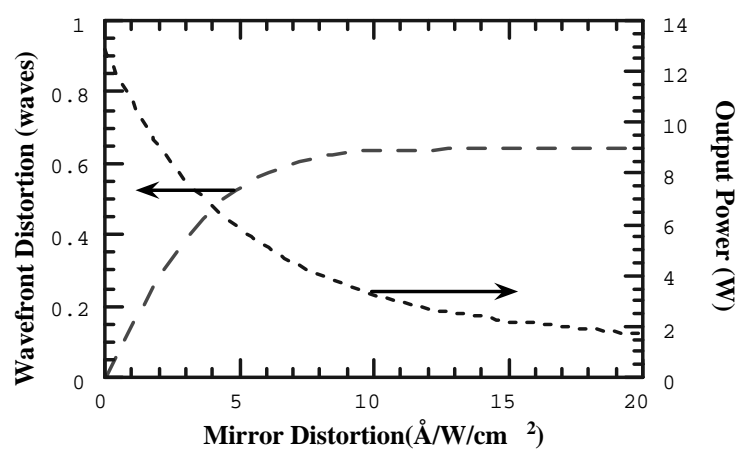

Fig. 1. Wavefront distortion and output power versus the mirror distortion per unit intensity for a UV unstable ring resonator with scraper output coupling. The shape of the distortion was assumed to follow the mode shape. 


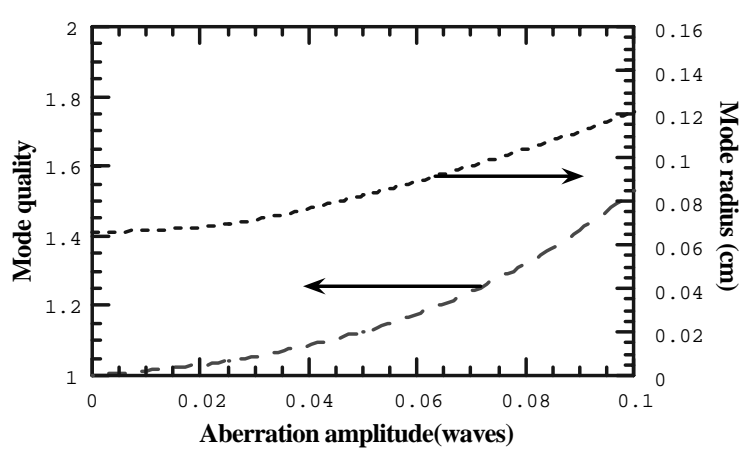

Fig. 2. Mode quality $M^{2}$ and waist spot radius as a function of Gaussian aberration added to both mirrors. The total wavefront distortion will be four times this value.

One can also do a simple Fox and Li type analysis of the with a phase error added to the cavity. This was done using the commercial code PARAXIA for a cavity with parameters of the IR Demo FEL operating at $3 \mu \mathrm{m}$. The mode quality factor $M^{2}$ and the waist spot size increase as a function of the Gaussian mirror distortion amplitude are shown in Fig. 2. Clearly a distortion in the mirror of $1 / 10$ of a wave is very deleterious to mode quality and should strongly affect the laser gain. On the other hand, a distortion of less than $1 / 20$ wave should have quite a small effect on the laser. Note that the fact that the cavity is nearly concentric makes the total aberration worse. The aberrations from each of the two mirrors add linearly due to the degeneracy of the cavity. For a smaller magnification, the mirror distortion induced aberration will be larger (see Eq. (4)) but the aberration from the two mirrors will not necessarily add linearly because the phase advance may alter or even reverse the wavefront distortion after propagating to the other mirror.

Another question to answer is how much the Rayleigh range can change before the gain is greatly reduced. For a system like the IR demo the answer is that the Rayleigh range must increase by a factor of four to decrease the gain by a factor of two. Note that, for this large a change in the Rayleigh range, the aberration will not necessarily be negligible. For a smaller magnification or in the transient regime the aberration will almost certainly dominate the reduction in laser gain.

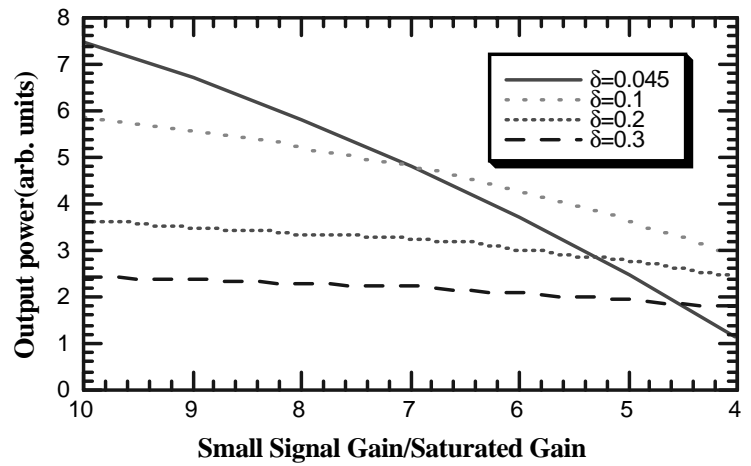

Fig. 3. Output power versus the ratio of the small signal gain to the saturated gain for four different cavity length detunings. A detuning of 0.045 corresponds to the maximum in the detuning curve for a gain ratio of 10 . Though the power is much higher for small detuning and high gain, it is not as high when the gain drops.

Finally, one must remember that it is the saturated gain that is important in determining the output power. The dependence of the saturated gain on the small signal gain is a complicated function of the cavity length detuning and the gain-to-loss ratio. If one assumes the cold cavity parameters of the IR Demo and uses Dattoli's approximation for the saturated gain versus the gain-to-loss ratio [7], one finds the curves in Fig. 3 for the output power versus the ratio of the small signal gain to the saturated gain. One can see that the power is much more sensitive to the ratio near the synchronous point than further out in the detuning curve.

\section{Experimental results}

In long pulse lasing measurements using a set of rather lossy $3 \mu \mathrm{m}$ mirrors (absorption for each mirror estimated at $0.4 \%$ ) we found the power curves shown in Fig. 4. The pulse length in each curve is $200 \mathrm{~ms}$. The laser was operated at the peak of the detuning curve at $3.1 \mu \mathrm{m}$. The lower curve is for $18.7 \mathrm{MHz}$ operation and the upper curve is with all the same parameters but with $37.4 \mathrm{MHz}$ operation. In the absence of mirror distortion, the power should have been independent of time for the two curves and equal to the initial power level. 


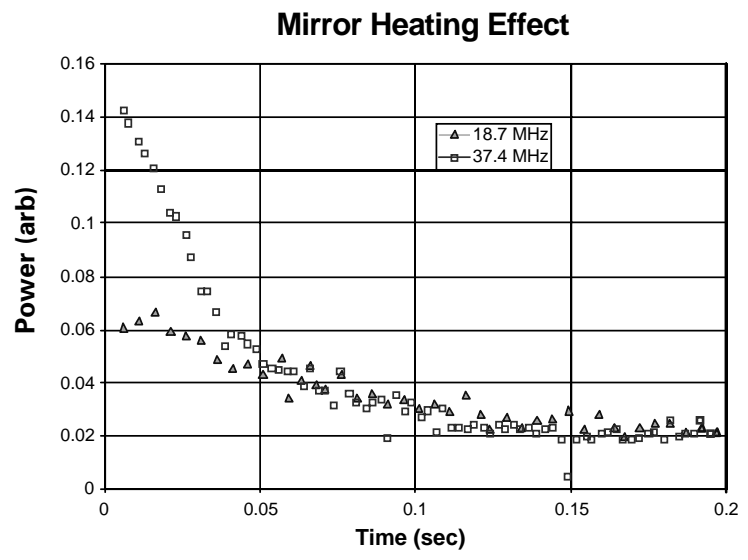

Fig. 4. Power versus time for long single pulse operation. The two curves are for the same laser and accelerator settings except that the repetition rate of the two differs by a factor of two.

Instead, the power for both cases falls rapidly and is the same after $50 \mathrm{~ms}$. This implies that the laser efficiency allowed at $37.4 \mathrm{MHz}$ is exactly half that allowed at $18.7 \mathrm{MHz}$. Note that the characteristic time for these mirrors from Eq. (5) is $0.33 \mathrm{~s}$, so the behavior seen is definitely occurring in the transient regime. Also note that the behavior is qualitatively consistent with the behavior seen in Fig. 1. As noted above, the data in Fig. 1 can be regarded as the power per micropulse train as a function of the repetition rate. One expects the power per micropulse to drop by a factor of two when the repetition rate is doubled. This means that the power should be independent of the repetition rate when the distortion is high enough. Also note that the initial slope of the curve is proportional to the repetition rate. This implies that the power reduction is due to thermal loading, which is linear in the initial power.

When the response seen in Fig. 7 is analyzed using the equations in Section 4, one finds that the absorption required for the aberration amplitude to reach $\frac{1}{10}$ of a wave in $30 \mathrm{~ms}$ (the time it takes for the experimental power to drop by a factor of two) is $2.8 \%$. The reflectivity of the high reflector is $99.6 \%$, so linear absorption this high is not possible. This seems to indicate that some nonlinear absorption mechanism is present in the coatings of these mirrors.

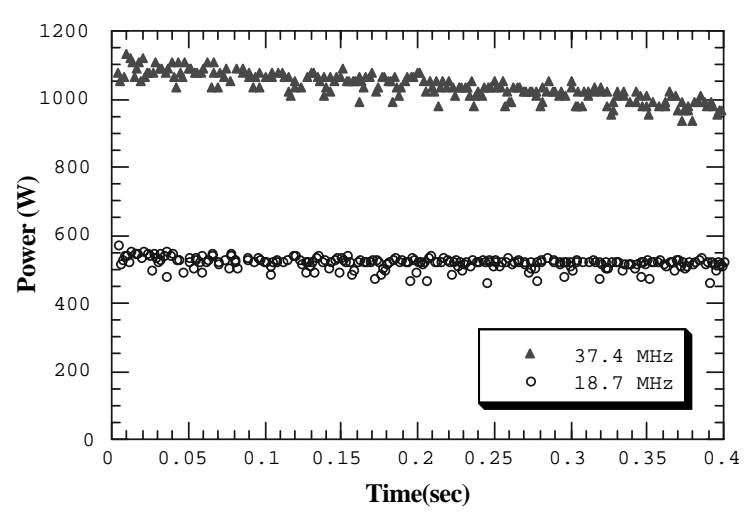

Fig. 5. Power versus time for $400 \mathrm{~ms}$ macropulses for two different micropulse repetition rates. The resonator used $3 \mu \mathrm{m}$ mirrors with low loss mirror coatings.

Power versus time curves for two $400 \mathrm{~ms}$ pulses using a different set of $3 \mu \mathrm{m}$ mirrors is shown in Fig. 5. The behavior is dramatically different from that shown in Fig. 4. The power falloff is linear over the entire macropulse and is quite small. Some of the falloff may be due to mirror steering effects caused by the mirror heating. The coherent harmonics were seen to move sideways during the long pulse. Clearly, no transient behavior is seen since the changes in power are small during the characteristic time for the mirror of $0.3 \mathrm{~s}$.

When similar data are taken for $\mathrm{CaF}_{2}$ mirrors, whose characteristic time is $2 \mathrm{~s}$, we find the behavior seen in Fig. 6. Here the falloff is more dramatic but the fall-time is comparable to the characteristic time and one expects that the characteristic time will be reduced as the mirrors heat up. Thus, the behavior seen cannot really be classified as occurring in the transient regime but must be simulated using a self-consistent timedependent theory.

Curves similar to those in Fig. 6 were taken as a function of cavity length detuning. We expected that the falloff in the power, at least on a relative scale, would go down with increasing cavity length detuning. We fit the power versus time at the start of the long pulse to a line of the form $P=P_{0}(1-b t)$ and plotted the value of $b$ versus the detuning. This is shown in Fig. 7. The behavior is exactly opposite to what one would 


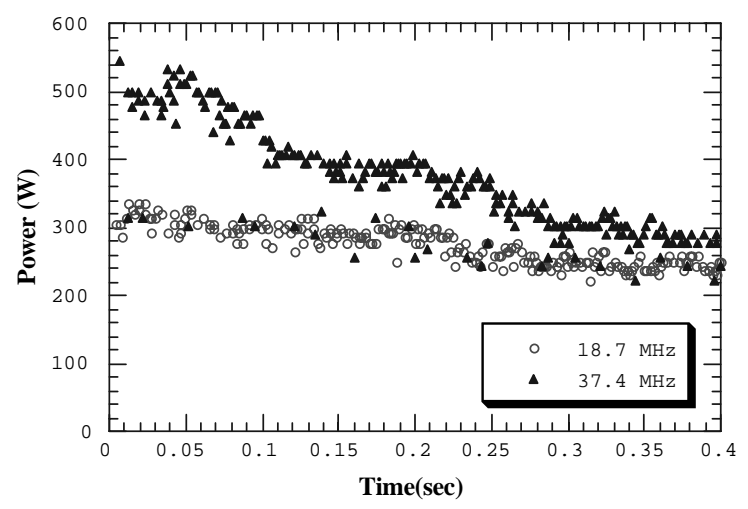

Fig. 6. Power versus time for $400 \mathrm{~ms}$ macroupulses. The FEL was operated at $5 \mu \mathrm{m}$ and used $\mathrm{CaF}_{2}$ mirrors with coatings similar to those on the sapphire mirrors used in Fig. 5.

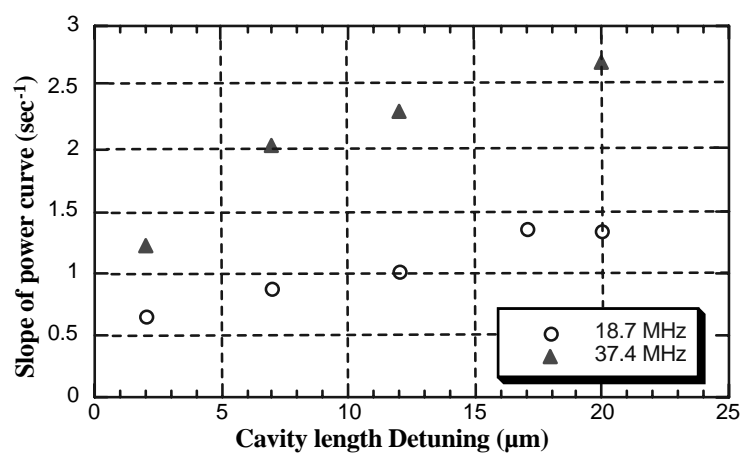

Fig. 7. Relative slope of the power versus time as a function of cavity length detuning for two different micropulse repetition rates using the $5 \mu \mathrm{m}$ resonator optics.

naively expect. The effect seems to be due only to the saturated gain in the absence of mirror distortion and not at all dependent on the small signal gain.

\section{Conclusions}

Though the transient response agrees qualitatively with the experimental results, the quantita- tive agreement is not as good. The only behavior that was clearly in the transient regime was the lossy $3 \mu \mathrm{m}$ mirrors. The other mirrors showed behavior consistent with adiabatic heating effects. The cavity length detuning dependence is puzzling and needs further study using a more complete four-dimensional FEL model. The transient behavior also needs more study with a self-consistent time-dependent model.

\section{Acknowledgements}

Many thanks to all the technical staff at Jefferson Lab who helped make this work possible. This work was supported by the U.S. Department of Energy under contract DE-AC05-84-ER40150, the Office of Naval Research, the Commonwealth of Virginia, and the Laser Processing Consortium.

\section{References}

[1] S.V. Benson, et al., Nucl. Instr. and Meth. A 407 (1998) 401.

[2] S.V. Benson, G.R. Neil, M. Shinn, in: S. Basu, S.J. Davis, E.A. Droko (Eds.), Gas, Chemical and Electrical Laser and Intense Beam Control and Applications, SPIE 3931, 2000, p. 243.

[3] C.L. Bohn, et al., Performance of the accelerator driver of Jefferson Laboratory's free-electron laser, Proceedings of the 1999 Particle Accelerator Conference, New York, 1999, p. 2450.

[4] N.N. Rykalin, Y.L. Krasulin, Sov. Phys. Dokl. 163 (1966) 87 (English Transl. 10 (1966) 659).

[5] G. Arfken, Mathematical Methods for Physicists, Section 5.11, Academic Press, New York, NY, 1970.

[6] J.C. Goldstein, B.D. McVey, Thermal distortion limits on the performance of XUV free-electron lasers configured with a multifacet ring resonator, High Heat Flux Engineering, SPIE 1739, 1992.

[7] G. Dattoli, P.L. Ottaviani, Logistic equation, FEL dynamics and self induced harmonic generation, presented at the 20th International Free-electron Laser Conference, Williamsburg, VA, August 1998. 